\title{
Power-Aware Virtual Network Function Placement and Routing using an Abstraction Technique
}

\author{
Amir Varasteh $^{\dagger}$, Marilet De Andrade ${ }^{\star}$, Carmen Mas Machuca ${ }^{\dagger}$, Lena Wosinska ${ }^{\star}$, and Wolfgang Kellerer ${ }^{\dagger}$ \\ $\dagger$ Chair of Communication Networks, Department of Electrical and Computer Engineering, \\ Technical University of Munich, Germany \\ Email: \{amir.varasteh, cmas, wolfgang.kellerer\}@tum.de \\ * Optical Networks Lab., KTH Royal Institute of Technology, Stockholm, Sweden \\ Email: \{marilet, wosinska\}@kth.se
}

\begin{abstract}
The Network Function Virtualization (NFV) is very promising for efficient provisioning of network services and is attracting a lot of attention. NFV can be implemented in commercial off-the-shelf servers or Physical Machines (PMs), and many network services can be offered as a sequence of Virtual Network Functions (VNFs), known as VNF chains. Furthermore, many existing network devices (e.g., switches) and collocated PMs are underutilized or over-provisioned, resulting in low powerefficiency. In order to achieve more energy efficient systems, this work aims at designing the placement of VNFs such that the total power consumption in network nodes and PMs is minimized, while meeting the delay and capacity requirements of the foreseen demands. Based on existing switch and PM power models, we propose a Integer Linear Programming (ILP) formulation to find the optimal solution. We also propose a heuristic based on the concept of Blocking Islands (BI), and a baseline heuristic based on the Betweenness Centrality (BC) property of the graph. Both heuristics and the ILP solutions have been compared in terms of total power consumption, delay, demands acceptance rate, and computation time. Our simulation results suggest that BIbased heuristic is superior compared with the BC-based heuristic, and very close to the optimal solution obtained from the ILP in terms of total power consumption and demands acceptance rate. Compared to the ILP, the proposed BI-based heuristic is significantly faster and results in $22 \%$ lower end-to-end delay, with a penalty of consuming $6 \%$ more power in average.
\end{abstract}

Keywords-Power optimization, Virtual Network Functions, VNF Chain.

\section{INTRODUCTION}

Network Function Virtualization (NFV) aims to tackle the limitations of hardware network functions [1] [2]. In this platform, network function implementations have evolved by running their software over virtualized general purpose hardware. These functions are known as Virtual Network Functions (VNFs). In the NFV architecture, a commercial off-the-shelf Physical Machine (PM) can host several Virtual Machines (VMs), each VM implementing a network function with special software programs. NFV can bring many benefits to the telecommunication networks: openness of platforms, scalability and flexibility, performance improvement, and also cost reductions [2].

In modern telecommunication, with the growth of cloud computing and virtualization technologies, network providers start deploying their network services using VNFs. A service is usually composed of various VNFs (e.g., firewall, WAN optimizer, Network translation service) based on customer demands. This ordered sequence of VNFs form a service function (VNF) chain [1]. However, effective deployment and resource allocation of VNF chains is a complex, yet important challenge to overcome. Especially, power consumption of these networks has always been a crucial issue [3]. Bolla et al. [4] show the aggressive trend of power consumption increase in the networks operated by the major telecom operators worldwide (e.g., AT\&T, Verizon). The high power consumption of these networks along with their high rate of growth and total carbon footprint have made it inevitable to apply Green Computing techniques and reduce the networks power consumption and guarantee the overall network scalability and sustainability.

Many studies have shown a massive waste of power consumed by idle or under-utilized devices. On one hand, PMs that are utilized by VNF instances are not power-proportional, since they consume more than $50 \%$ of their maximum power when they are in idle state [5]. Therefore, to provide powerefficiency, one solution is to increase PMs resource utilization, which leads to using lower number of active PMs. On the other hand, network devices are also an undeniable powerconsuming part of telecommunication networks [6]. Similar to the computation resources, network resources are also usually over-provisioned to support the maximum traffic. However, their utilization rarely reaches the peak network capacity [7]. Consequently, idle networking devices are not power-efficient, since an idle network switch can consume up to $90 \%$ of the peak power consumption [6]. Thus, minimizing the number of active network devices can also contribute to the overall power-efficiency.

Keeping in mind the above observations, in this work, we study the joint power-aware VNF chain placement and routing problem. The main goal is to minimize the amount of computation (i.e., PMs) and network (i.e., switches and links) resources required to realize VNF chains while meeting end-to-end delay requirements. To do so, we proposed the optimal power-aware placement of VNFs using an Integer Linear Programming (ILP) formulation. Due to the complexity of the ILP, we also proposed an efficient heuristic based on the Blocking Island (BI) resource abstraction paradigm.

The main contributions of this paper are: $(i)$ a solution to the VNF chain placement problem using ILP (ii) Design of an efficient heuristic for joint power-aware VNF chain placement and routing using the BI resource abstraction technique, and (iii) a new heuristic based on Betweenness Centrality (BC) property of graph [8], which is used as baseline. The rest of the paper is organized as follows: Section II reviews the related works. Section III presents the system model and problem formulation. Then, the two proposed heuristics are introduced in Section IV, followed by the performance evaluation in Section V. Finally, Section VI concludes the paper.

\section{RELATED WORK}

A comparison of different power aware placement solutions with respect to the proposed one has been depicted in Table 
TABLE I: Characteristics considered in the related work

\begin{tabular}{cccccc}
\hline Works & PM Power & Network Power & Delay & Functions Order & Instance Sharing \\
\hline$[12][17]$ & $\checkmark$ & $\times$ & $\times$ & $\times$ & $\times$ \\
\hline$[9][10]$ & $\checkmark$ & $\checkmark$ & $\times$ & $\times$ & $\times$ \\
\hline$[11]$ & $\checkmark$ & $\times$ & $\checkmark$ & $\times$ & $\times$ \\
\hline$[18]$ & $\times$ & $\checkmark$ & $\times$ & $\times$ & $\times$ \\
\hline$[19][20]$ & $\checkmark$ & $\times$ & $\checkmark$ & $\checkmark$ & $\checkmark$ \\
\hline$[21][22][23]$ & $\checkmark$ & $\checkmark$ & $\times$ & $\checkmark$ & $\checkmark$ \\
\hline$[24]$ & $\checkmark$ & $\times$ & $\checkmark$ & $\times$ & $\checkmark$ \\
\hline$[25]$ & $\checkmark$ & $\times$ & $\times$ & $\checkmark$ & $\checkmark$ \\
\hline$[26]$ & $\checkmark$ & $\times$ & $\checkmark$ & $\checkmark$ & $\checkmark$ \\
\hline Our approach & $\checkmark$ & $\checkmark$ & $\checkmark$ & $\checkmark$ &
\end{tabular}

I. Power-aware VM placement has been widely studied in the cloud computing environment [9]-[12]. Surveys on VM placement techniques can be found in [13], [14]. Most of the VM placement studies are focused on minimizing either PM or network power consumption. However, VNF chain placement strategies differ from VM placement solutions in mainly ways. Firstly, the VNF chain placement problem, considers VNF chains, which requires a specific ordered set of VNFs, which is not the case in VM placement problems. Additionally, VNF chain placement problem can be considered as two NP-hard problems: Virtual Network Embedding, and Location-Routing Problem [15], which is not the case for VM placement. Thus, these specific characteristics and constraints has made the VNF placement problem more complex. A comprehensive survey on VNF chain placement is presented in [16].

Several studies investigated the VNF placement problem with different objectives (e.g., demand delay and cost). However, there are only a few studies that have focused on minimizing the energy/power consumption. El Khoury et al. [24] formulated the problem to allocate and schedule traffic flows with deadlines to VNFs while minimizing the total PM power consumption in the network. Notably, in their scenario, VNF instances are already placed in the network. Kim et al. [19] proposed an energy-aware VNF chain placement and reconfiguration algorithm based on Genetic Algorithm to minimize the PMs power consumption, considering QoS requirements in terms of latency. Pham et al. [20] formulated an optimization problem to minimize the joint operational and network traffic cost. Their solution aimed at deploying as fewer number of PMs such that the communication cost between them was optimized. They used a sampling-based Markov approximation combined with matching theory to solve the combinatorial NPhard problem in a quick way. Huin et al. [21] proposed an ILP to minimize the networking power consumption named GreenChains. Yang et al. [22] studied VNF chain placement in datacenters. They provided an algorithm named Merge$R D$ to save power in servers and network switches, and also reduce the transmission delay. Marotta et al. [26] presented a joint resource and flow routing assignment mathematical model in order to minimize the power consumption of the hosting PMs and network devices. They used Robust Optimization theory to cope with data uncertainty in vEPC mobile networks. Actually, they defined a trade-off between power-efficiency, robustness, and the ability of the network to cover the data uncertainty. Eramo et al. [25] went a step further and proposed a server consolidation approach to achieve energy efficiency by turning off as many PMs as possible when the traffic density decreased. They formulated an optimization problem and proposed heuristics for online and offline cases, where the objective function is minimizing total PMs and VM migration energy consumption. Finally, Soualah et al. [23] focused on minimizing the total PMs and network switches energy consumption. They formulated VNF placement and chaining problem by using the Monte Carlo Tree Search method and compared their solution to several other works.

However, to the best of our knowledge, there is no work that tackled the VNF chain placement problem by jointly considering PMs and network power consumption, while satisfying end-to-end delay requirements. Furthermore, we apply the BI abstraction paradigm to enhance the VNF placement.

\section{System Model AND Problem Formulation}

We represent the network as a bidirectional graph $G(N, L)$, where $N$ is the set of nodes (network switches) and $L$ is the set of links in the network. In this design problem, every node is eligible to have a collocated PM that is able to host VNFs. Every PM has a set of resources $R$, which consist of: CPU, memory and storage. A set of demands $G$ is given, each demand $g$ been defined as:

$$
g\left(v_{s, g}, v_{d, g}, D_{g}, B_{g}, c_{g}\right)
$$

where $v_{s, g}$ is the source node, $v_{d, g}$ is the destination node, $D_{g}$ is the end-to-end delay, $B_{g}$ is the required bandwidth capacity and $c_{g}=\left\{f_{1} \rightarrow f_{2} \rightarrow \ldots \rightarrow f_{\left|c_{g}\right|}\right\}$ is the a set of ordered service functions, that is, the VNF chain $c_{g}$ of that demand. VNF chains are modelled as a virtual network represented as a graph $G\left(V_{g}, E_{g}\right)$, where $V_{g}$ is the set of virtual nodes for demand $g$, and $E_{g}$ is the set of virtual links for demand $g$. The virtual nodes represent the functions (belonging to the given set of functions $F$ ) and endpoints of demand $g$, and the virtual links interconnect the virtual nodes following the order of that chain. In this way, $G\left(V_{g}, E_{g}\right)$ has to be mapped over $G(N, L)$ such that the demand $g$ requirements are guaranteed while minimizing the consumed power. The ingress and egress virtual nodes match the physical source and destination nodes in the substrate network.

A function $f$ can be reused by different demands as long as its maximum processing capacity [in $\mathrm{Mb} / \mathrm{s}$ ] $B_{f}$ is not surpassed. Otherwise, a new VNF should be placed in an active PM if it has enough resources. Finally, if active PMs do not have enough resources, a new PM must be activated. These three alternatives have different impact on the power consumption as introduced in the next section.

\section{A. Power Model}

The following switch and PM power models are considered. The switch power consumption is defined as the sum of the power consumed regardless of the traffic load $P_{s s}$ and power proportional to the active ports [27], that is:

$$
P_{s w}=P_{s s}+P_{p} N_{p}
$$

where $P_{p}$ is the amount of power consumption of each active port, and $N_{p}$ is total number of active ports in a switch.

Considering that the active physical link $l_{i, j} \in L$ from node $i$ till node $j$ requires two ports, the total network power consumption can be expressed as:

$$
P_{n e t}^{T}=P_{s s} \sum_{i \in N} y_{i}+2 P_{p} \sum_{i j \in L} l_{i, j}
$$

where $l_{i, j}$ is a binary variable that is equal to 1 if the physical link between node $i$ and $j$ is active. Notably, we assume a single link between each pair of nodes. Additionally, in the above equation, $y_{i}$ is a binary variable indicating if switch $i$ is active.

Since the CPU is the most significant power consumer in a server [28]-[30], the power consumption model for the PM 
is based on its CPU utilization, which can be calculated using the following equation [31]:

$$
P_{p m}=P_{s m}+\left(P_{m m}-P_{s m}\right) \times \theta_{c p u}
$$

where $P_{s m}$ corresponds to the static power consumption of the PM (i.e., the consumed power when its utilization is $0 \%), P_{m m}$ is the power consumption of the PM in its maximum CPU utilization and $\theta_{C P U}$ represents the CPU utilization. $\theta_{C P U}$ can be calculated as the ratio between the total CPU resources required and the available $\mathrm{CPU}$ resources available in the PM: $\theta_{C P U}=C_{f, r} / C_{i, r}^{T}$, where $r$ represents CPU resource type. Thus, the total power consumption of PMs in the network $P_{p m}^{T}$ can be expressed as follows:

$$
P_{p m}^{T}=\sum_{i \in N}\left(P_{s m} x_{i}+\left(P_{m m}-P_{s m}\right) \sum_{f \in F, r \in R} \frac{C_{f, r}}{C_{i, r}^{T}} z_{i, f}\right)
$$

where $x_{i}$ is a binary variable indicating if the PM $i$ is active, and $z_{i, f}$ is an integer variable representing the number of instances of function $f$ placed on the PM at node $i$.

\section{B. ILP Formulation}

Based on our previous work [32], we mathematically formulate the joint power-aware VNF chain placement and routing problem using an ILP in order to obtain the optimal solution. The goal of this work is to optimally place VNF chains, such that overall power consumption of network switches, links, and PMs is minimized, while meeting end-toend delay requirements. Therefore, the objective function is:

$$
\text { Minimize }\left(P_{n e t}^{T}+P_{p m}^{T}\right) \text {. }
$$

In addition to Eq. (2) and (4), the presented objective function must satisfy a number of constraints which are as follows:

$$
\begin{aligned}
& \sum_{f \in F} C_{f, r} z_{i, f} \leq C_{i, r}^{T}, \forall i \in N, \forall r \in R \\
& \sum_{g \in G} B_{g} u_{i, f, g} \leq B_{f} z_{i, f}, \forall i \in N, \forall f \in F \\
& \sum_{k l \in E_{g}} \sum_{g \in G} B_{g} w_{i j, k l, g} \leq B_{i, j}, \forall i \in N, \forall j \in N \\
& u_{i, f, g} \leq z_{i, f}, \forall i \in N, \forall f \in F, \forall g \in G, \\
& \sum_{i \in N} \sum_{f \in F} D_{f} u_{i, f, g}+\sum_{i j \in L} \sum_{k l \in E_{g}} D_{i, j} w_{i j, k l, g} \leq D_{g} \\
& , \forall g \in G, \\
& \sum_{i j \in L} w_{i j, k l, g}-\sum_{j i \in L} w_{j i, k l, g}=u_{i, k, g}-u_{i, l, g} \\
& , \forall i \in N, \forall k \in V_{g}, \forall l \in V_{g}, \forall g \in G, \\
& u_{i, f, g}=1 \quad, i f \quad i=f=v_{s, g}, \quad \forall g \in G, \\
& u_{i, f, g}=1 \quad, i f \quad i=f=v_{d, g}, \quad \forall g \in G .
\end{aligned}
$$

Eq. (7) indicates that the resources $C_{f, r}$ used by the functions placed in the PM that is connected to node $i$ must not exceed the available resources in it $C_{i, r}^{T}$. In Eq. (8), the capacity requirements of all demands served by function $f$ in the PM in node $i$ is limited to the processing capacity of function $f$, where $u_{i, f, g}$ is a binary variable which equals to 1 if function $f$ for demand $g$ is placed in PM $i$. Equation (9) states that the bandwidth required by all demands served by link $(i, j)$ will not be larger than the capacity of the link $(i, j)$, noted as $B_{i, j}$, where $w_{i j, k l, g}$ is a binary variable that equals to 1 if the physical link $(i, j)$ is used by the virtual link $(k, l)$ of demand $g$. In Eq. (10), it is stated that demand and function mapping must match. In Eq. (11), the total delay in the path, due to processing delay $D_{f}$ of all present functions $f$ in the PMs and the propagation delays $D_{i, j}$ in the physical links provided for demand $g$, is limited to the required end-to-end delay $D_{g}$ for demand $g$. The flow conservation law constraint is expressed in Eq. (12), which states that for each network switch $i$, the

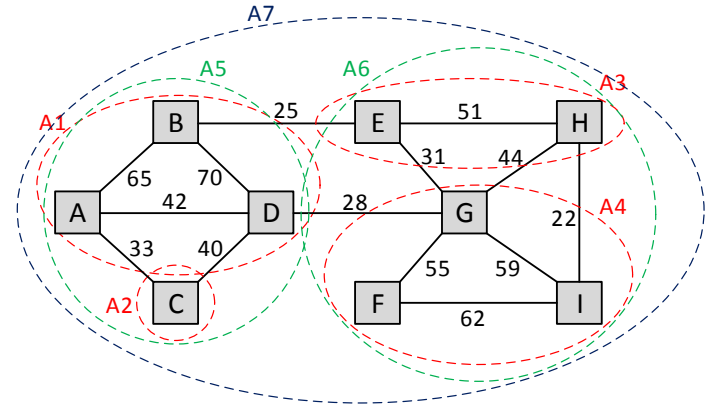

Fig. 1: An example of BIG, when $\beta$ values are 50, 40, and 30. A1-A4 are 50-BIs, A5-A6 are 40-BI, and A7 is 40-BI. Link weights are their available bandwidth.

difference of all outgoing and incoming physical links that are used for the virtual link between virtual nodes $k$ and $l$, and demand $g$ must be equal. Eq. (13) and (14) map the endpoints of the demand to the PMs in the substrate network.

Finally, let us introduce three indicator variables, defined in Eq.(15), Eq. (16), and Eq. (17) respectively. These variables control the operation status (i.e., online or offline) of physical links, PMs, and network switches, respectively. Note that $\Psi$ is defined as a large positive number.

$$
\begin{aligned}
& \sum_{k l \in E_{g}} \sum_{g \in G} w_{i j, k l, g} \leq l_{i, j} \times \Psi, \quad \forall i \in N, \forall j \in N, \\
& \sum_{j \in N}\left(l_{i, j}+l_{j, i}\right) \leq y_{i} \times \Psi, \quad \forall i \in N \\
& \sum_{f \in F} z_{i, f} \leq x_{i} \times \Psi, \quad \forall i \in N
\end{aligned}
$$

\section{Proposed Heuristic}

In order to cope with the ILP scalability issue, we present an effective heuristic approach, which consists of two main steps: 1) Network abstraction using the Blocking Island (BI) technique; and 2) Power-aware joint VNF chain placement and routing.

\section{A. Blocking Island Paradigm}

The BI technique, which is derived from Artificial Intelligence, is a resource abstraction method to represent the availability of resources (in this paper, bandwidth) in a graph [33]. The goal is to abstract the network into different subgraphs (for different $\beta$ values).

$\beta$-BI Definition: [33] A $\beta$-BI for node $x$ is the set of all nodes of the network that can be reached from $x$ using link(s) with at least $\beta$ available bandwidth.

Let us consider the network depicted in Fig. 1 with 9 nodes and 14 links. $A 1$ is the $50-\mathrm{BI}$ for node $B$ (i.e., $\beta=50$ ) since all the nodes of $A 1$ can reach $B$ and any other node of $A 1$ with a path with at least 50 units of bandwidth. Since node $C$ can be connected with at most with 40 units of bandwidth, it does not belong to $A 1$. The $\beta$-BI for a give node $x$ can be obtained by using a fast greedy algorithm, called $\beta$-Blocking Island Search (BIS) [33], which its worst-case complexity is linear in $O(|L|)$ where $L$ is the set of links in the graph.

For each $\beta$ value and the network graph, different $\beta$-BIs can be found, which can be represented as a $\beta$-BI Graph (BIG). For example, Fig. 2(a) depicts the 50-BIG for the Fig. 1 graph. The abstract nodes are the different $\beta$-BIs and the abstract links the network links interconnecting each pair of islands with the maximum bandwidth. Furthermore, for a given set of 


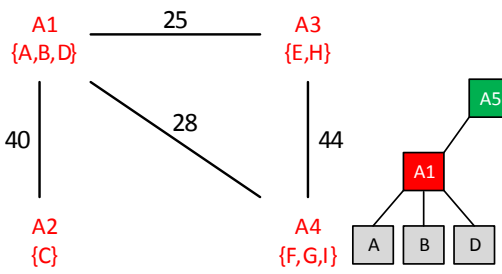

(a)

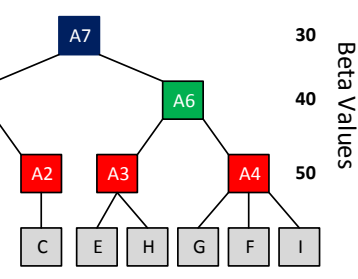

(b)
Fig. 2: (a) 50-BIG and (b) BIH tree from network in Fig. 1

$\beta$ values $\mathcal{B}=\left\{\beta_{1}, \beta_{2}, \ldots, \beta_{|\mathcal{B}|}\right\}$, an overall network abstraction can be presented (so-called Blocking Island Hierarchy (BIH)). BIGs can be decomposed as a vertical tree structure in decreasing order of $\beta$ s as shown in Fig. 2(b) for our example. This abstraction tree can reflect the real-time state of the available network bandwidth.

\section{B. BI-Based Heuristic}

For a given demand defined by $\mathrm{Eq} 1$, the $\mathrm{BI}$ paradigm first looks for $\beta$-BIs with $\beta \geq B_{g}$ containing both source and destination. If there are not any, the demand is rejected, since it can not be assigned, otherwise, the algorithm will proceed. Hence, the search space and time complexity decreases significantly. However, BIH should be updated every time a new demand is served by recomputing the BIs involved in that demand. This re-computation is performed in terms of merging or splitting operations which their complexities are only $O(n)$ or $O(l)$ [33], where $n$ and $l$ denote the number of involved nodes and links on the new demand, respectively. Besides, experimental results show that despite updating costs, BI can greatly improve overall computation efficiency [34]. Since the VNF chain placement and routing problem is NP-complete, the ILP formulation presented in Section III-B is not scalable. The BI paradigm is proposed as an efficient and fast solution. The heuristic algorithm consists of four main steps, which is presented in Algorithm 1. These steps are:

Step 1: Given the network graph $G(N, V)$ and a set of $\beta$ values $\mathcal{B}=\left\{\beta_{1}, \beta_{2}, \ldots, \beta_{|\mathcal{B}|}\right\}$, the $\mathrm{BIH}$ is built (line 1 ).

Step 2: For every demand defined as Eq. (1), a BI is selected (selected $B I$ ) that contains the source and destination and $\beta \geq$ $B_{g}$ ) (line 3$)$. In this study, two BI selection approaches have been considered:

(i) Highest $\beta$-BI (HBI): In case several BI are available, the one with highest highest $\beta$ value in the BIH. By using this approach, the selected BI for hosting the request may contain less number of nodes. Thus, it can be expected that the probability of reusing VNFs decreases (i.e., more power consumption). Also, since HBI selects a BI with fewer nodes, fewer nodes should be checked for VNF chain placement. In this way, the time complexity is expected to reduce.

(ii) Lowest $\beta$-BI (LBI): In case several BI are available, the $\mathrm{BI}$ with the lowest $\beta$ value in the BIH is selected. With LBI, the selected BI may contain more nodes than HBI. Hence, it is expected that the VNF reuse probability increases (i.e. less power consumption) and also incurs in more time complexity. The performance of these approaches is analysed in Section V. Step 3: The joint power-aware VNF chain placement and routing algorithm tries to place the VNF instances and to find the route of this demand such that the total network and PM power is minimized. VNFs can be reused if they have enough
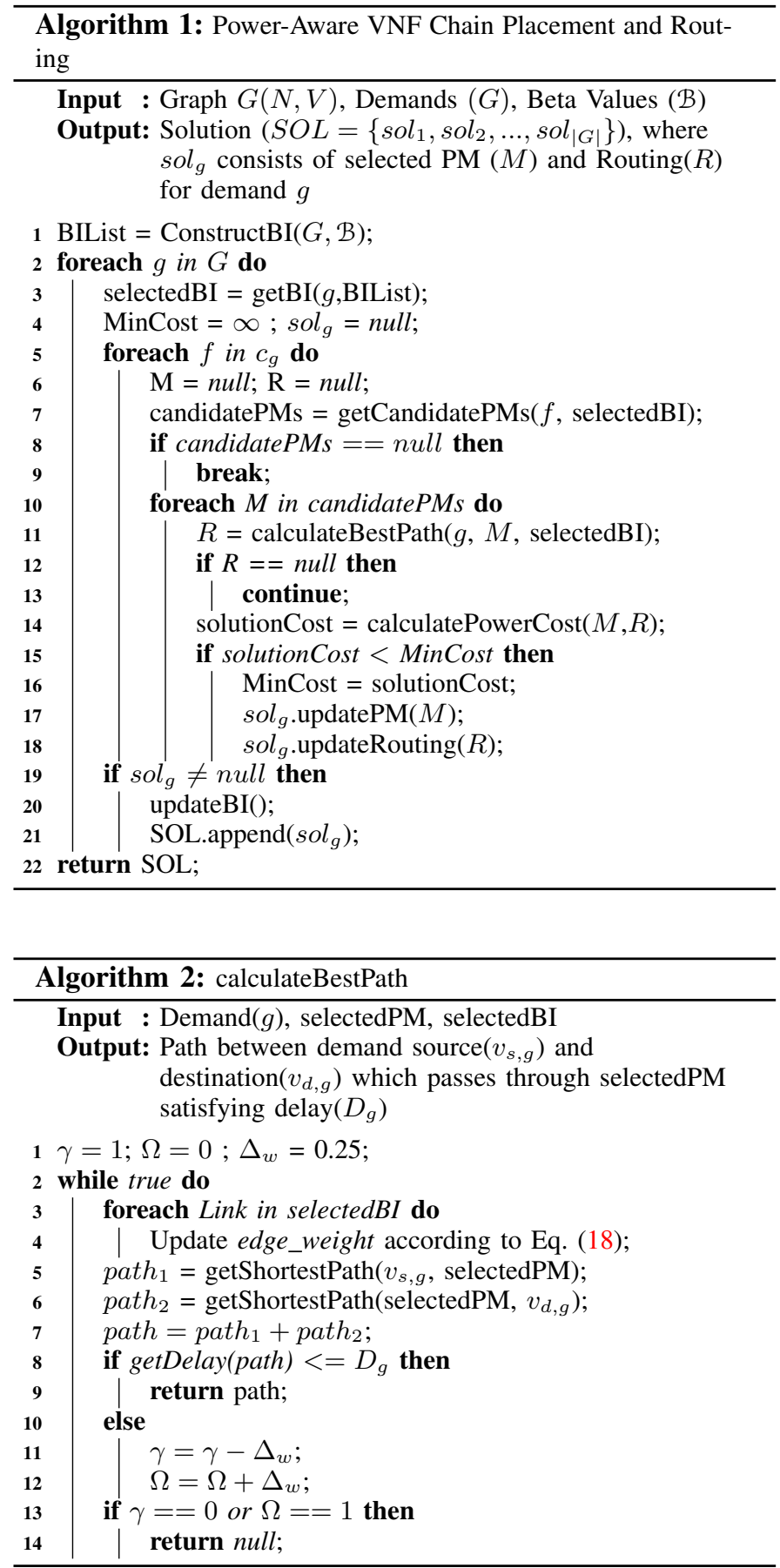

capacity or they can be replicated at a new server, which can be active or should be turn it on with an extra power consumption. This algorithm consists of two main parts which are:

Step 3.1: VNF Placement: As it can be seen in Alg. 1 lines 6-7, for every network function $f$ of the chain $c_{g}$, the PMs of selectedBI that can host function $f$ are found with getCandidateList. These candidatePMs PMs can be (i) PMs that already have an instance of function $f$ with enough processing capacity to accept $g$, (ii) PMs that are online and have enough available resources for creating a new instance of $f$, or (iii) PMs that are offline. Note that the power cost of the option is increasing from (i) to (iii). 
Step 3.2: Power-aware routing: It finds the path from demand source $v_{s, g}$ to its destination $v_{d, g}$ containing intermediate node candidateP $M$. The proposed power-aware routing algorithm is presented in Alg. 2. This algorithm considers an edge weight edge_weight, which is a weighted sum of normalized values of consumed power edge_power and delay edge_delay of the edge. The normalization of edge_power and $\bar{e} d g e \_$delay is calculated based on the maximum possible power consumption and maximum link delay in the network, respectively. The edge_Power value of link $(i, j)$ is calculated as $\left(1-\operatorname{state}\left(S_{i}\right)\right) \times \frac{1}{2} P_{s s}+\left(1-\operatorname{state}\left(S_{j}\right)\right) \times \frac{1}{2} P_{s s}+$ $\left(1-\operatorname{state}\left(l_{i, j}\right)\right) \times 2 P_{p}$ (see Eq. (2)), where $\operatorname{state}\left(S_{i}\right)$ and state $\left(L_{i, j}\right)$ are equal to 1 if the status of switch $i$ and link $(i, j)$ is online, respectively, otherwise 0 . Therefore, the expression of the edge's weight can be written as:

$$
\text { edge_weight }=(\gamma \times \text { edge_power }+\Omega \times \text { ed } \widehat{\text { ge_delay }}) \text {. }
$$

The weighting parameters $\gamma$ and $\Omega$ are set to highlight the relevance of power over delay. The initial value for $\gamma$ and $\Omega$ is set to 1 and 0 , but they will change gradually during the execution of the algorithm.

Once the edge weights are assigned, the algorithm tries to find a path from source to the selected $P M$ and from the selectedPM to the destination. The total path's delay is computed and compared with the demand's delay requirement $\left(D_{g}\right)$. If the path's delay is compliant with the requirement (i.e., $\leq D_{g}$ ), the path is accepted. Otherwise, the edge weights are updated according to a given $\Delta_{w}$ value, which allows changing weights $\gamma$ and $\Omega$. This iterations continue until $\gamma=0$ or $\Omega=1$ (delay becomes the only priority). Actually, we use these weights to increase the demand acceptance rate by lowering the power-efficiency priority in each iteration. Indeed, there is a trade-off between power-efficiency and demand acceptance rate, since reusing network resources can increase the delay, which can lead to demand rejection. Nevertheless, the algorithm can be tuned by determining bounds for $\gamma$ and $\Omega$ and also the value of $\Delta_{w}$ for different objectives.

After selectedPM is determined, the power consumption of the candidate solution can be calculated, which is the sum of the PM and network power (Eq. (4) and (2), respectively). The solution with the minimum cost is kept as potential final solution for function $f$. Notably, if there are two solutions with the same cost, we select the PM which is the closest one to the source. This will leave more PMs to explore on the shortest-path. This procedure continues until all PMs in the selected $P M$ list are examined.

Step 4: The affected BIs in the graph are updated (line 26) and add the selected PM $(M)$ and routing $(R)$ for demand $\mathrm{g}$ $\left(s o l_{g}\right)$ to the solution list $(S O L)$. The same method applies to all demands in the demand set $G$ (starting with Step 1). Finally, the solution for all demands (i.e., $S O L$ ) is returned and the algorithm terminates.

\section{Betweenness Centrality-Based Heuristic}

In order to evaluate the advantages of using BI paradigm, a second heuristic algorithm based on the betweenness centrality $(B C)$ property of a graph has been developed. The BC of a node $v[8]$ is defined as:

$$
g(v)=\sum_{s \neq v \neq t} \frac{\sigma_{s t}(v)}{\sigma_{s t}}
$$

where $\sigma_{s t}$ is the total number of shortest paths from $s$ to $t$ and $\sigma_{s t}(v)$ is the number of paths that pass through $v$. This BCbased algorithm first calculates the $\mathrm{BC}$ value for every node
TABLE II: Considered service function chains [21]

\begin{tabular}{ccccc}
\hline Service Type & VNF Chain & Bandwidth & Delay & $\%$ Traffic \\
\hline Web Service & NAT-FW-TM-WOC-IDPS & $100 \mathrm{kbps}$ & $500 \mathrm{~ms}$ & $18.2 \%$ \\
\hline VoIP & NAT-FW-TM-FW-NAT & $64 \mathrm{kbps}$ & $100 \mathrm{~ms}$ & $11.8 \%$ \\
\hline Video Streaming & NAT-FW-TM-VOC-IDPS & $4 \mathrm{Mbps}$ & $100 \mathrm{~ms}$ & $69.9 \%$ \\
\hline Online Gaming & NAF-FW-VOC-WOC-IDPS & $50 \mathrm{kbps}$ & $60 \mathrm{~ms}$ & $0.1 \%$ \\
\hline
\end{tabular}

in the graph. Then, for each demand and its shortest path, the algorithm tries to place the required VNFs on the nodes with higher $\mathrm{BC}$ value. According to the $\mathrm{BC}$ definition, a higher $\mathrm{BC}$ value means higher number of shortest paths that are passing through that node $v$ and hence, higher probability of $\mathrm{VNF}$ reuse, which can lead to power-efficiency in a network.

\section{Performance Evaluation}

In this section, the proposed heuristics are compared with the ILP solution. The comparison is done in terms of the power consumption, end-to-end delay, acceptance rate, BI selection and runtime. The proposed ILP model has been implemented using the Gurobi Optimizer 5.0 solver in Python. The BIbased and BC-based heuristics have been implemented in Java environment. The simulations were executed on a machine equipped with Intel Core i7-6700@3.40 GHz, 16 GB of RAM, running Windows 10 x64 OS.

The used network topology is the SNDLib Nobel Germany with 17 nodes and 26 links [35]. The capacity of each physical link was set to $1 \mathrm{Gbps}$ and their delay were determined proportional to their length considering optical fiber transmission. PMs are located at each node, each PM being able to host VNF instances. We assumed each PM is equipped with a 16-core processor. The power models for network and PM follow Eq. (2) and Eq. (4), respectively. The considered values are: switch power $P_{s s}=130 \mathrm{~W}$, link power $P_{p}=1 \mathrm{~W}$ [6], static PM power $P_{s m}=150 \mathrm{~W}$, and maximum PM power $P_{m m}=250 \mathrm{~W}$ [36].

The VNF chains described in [21] have been considered and summarized in Table II. These chains include different service types with specific ordered set of VNFs and different requirements in terms of bandwidth and delay. Different function types have been considered: Network Address Translation (NAT), Firewall (FW), Traffic Monitor (TM), WAN Optimization Controller (WOC), Video Optimization Controller (VOC), and Intrusion Detection System (IDS). In addition, network traffic is divided to these service types by a specific percentage (e.g., $11.8 \%$ for VoIP service type according to Table II). Using this information, different sets of demands can be generated. We uniformly distributed the source and destination nodes, and randomly assigned each demand to a service type based on the traffic percentages. All VNFs are assumed to have $200 \mathrm{Mb} / \mathrm{s}$ traffic processing capacity, and 10 ms of processing delay. We used $10 \mathrm{~ms}$ to put stress on the ILP and heuristic algorithms, according to large end-to-end service delay requirements in Table II. We also assumed that each function requires $4 \mathrm{CPU}$ cores to be able to operate. Notably, static $\beta$ values as $\mathcal{B}=\{900,700,500,300\}$ and LBI BI selection approach have been considered for the first simulations. Each simulation has been executed 30 times so that mean value and standard deviation can be plotted in the figures.

A. Power Consumption: The total power consumption results are compared in Fig. 3. It can be seen that the ILP can only solve small problem instances, while BI-based and BC- 


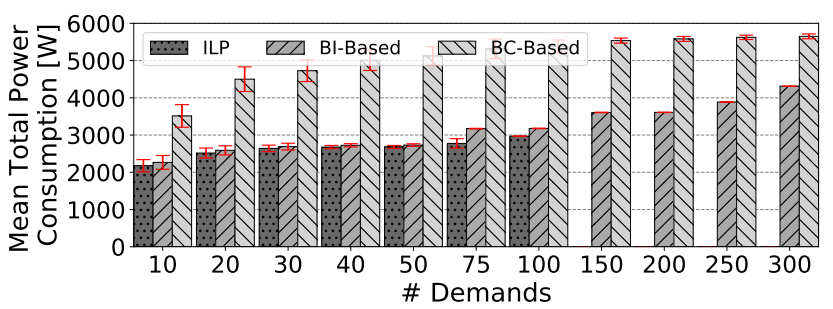

(a)
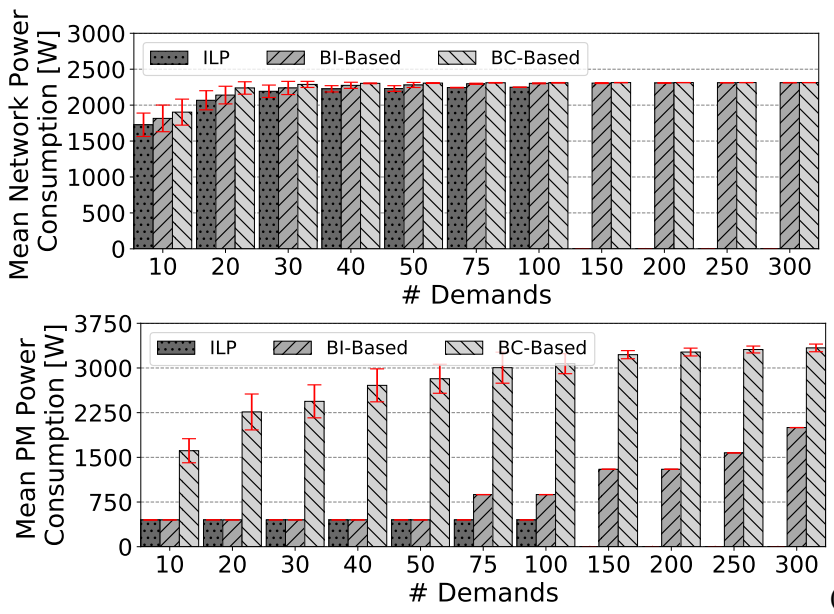

Fig. 3: Total, network, and PM power consumption comparison

based heuristic are more scalable. The results show that the total power consumption achieved by the BI-based algorithm is closer to the optimal solution than the BC-based algorithm. Also, the mean PM power consumption in BC-based algorithm is significantly higher than the BI-based and the optimal solution. That is because it selects the shortest path, instead of using longer but more power efficient paths like BI-based and ILP. It can also be observed that the network power consumption of the BI-based outperforms the BC-based algorithm, while it is close to the optimal solution. Also, we note that for more than 100 demands, the two heuristics consume almost the same amount of power for operating the networking part. This is because the network is overloaded with the demands.

B. End-to-End Delay: Fig. 4 presents the average total endto-end delay for all demands and compares this parameter for the three proposed approaches. It can be seen that the delay obtained by the BC-based algorithm is always lower than the one achieved by the BI-based one. The reason is the BIbased algorithm may select the longer path when allocating a demand (compared with the BC-based algorithm, which takes the shortest path). On the other hand, ILP model incurs higher delay than the two heuristics. This is because the ILP objective is to minimize total power consumption, so it reuses the resources as much as possible which adds delays due to a longer path. Thus, there is a trade-off between delay and

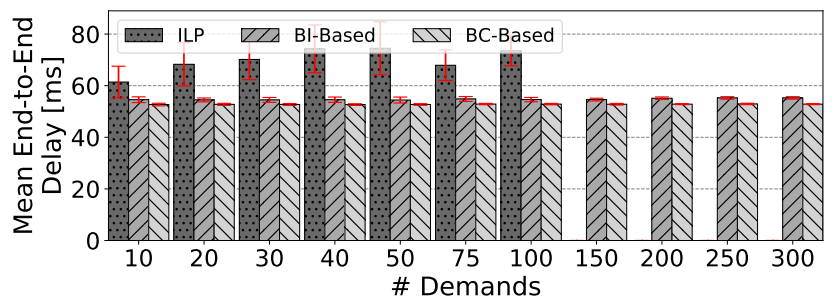

Fig. 4: Comparison of the average total end-to-end delay.

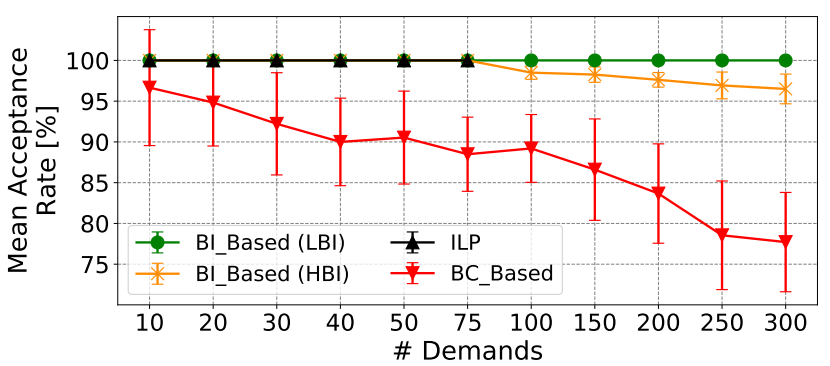

Fig. 5: Comparison of acceptance rates.

power consumption in this scenario.

C. Acceptance Rate: It is defined as the percentage of demands that have been hosted in the network. Fig. 5 compares the acceptance rate for the ILP model, BI-based, and BC-based heuristics. In all the simulations with different sets of demands, ILP and BI-based approaches are able to serve all the demands. However, the BC-based algorithm cannot host all the demands is focusing only on the shortest path to be able to use the $\mathrm{BC}$ property. Hence, for the 300-demand case, it is able to accept only $80 \%$ of the demands.

D. BI Selection: We compared the impact of the BI selection approaches LBI/HBI introduced in Section IV-B, which select the lower/highest possible $\beta$ value, respectively. The power consumption for both methods is depicted in Fig. 6. It can be observed that LBI is more power-efficient than HBI. The reason is that, according to the $\mathrm{BIH}$ tree, getting a lower $\beta$ value means a larger BI with more potential PMs and reusable resources. This fact can also lead to different demand acceptance rate. As it is shown in Fig. 5, the mean acceptance rate for HBI approach is lower than LBI. However, since there are potentially fewer nodes in the HBI approach, its computational-efficiency is higher than LBI due to the reduced search space.

E. Runtime: Since the computation time is critical for the demand setup times, the computation time of the three solutions have been compared for different number of demands. As it can be seen in Table III, the ILP runtime (given in seconds) is significantly higher than the two heuristics. In fact, it grows exponentially with increasing number of demands compared with a linear increase of both heuristics. Additionally, it can be seen that the runtime of the BI-based algorithm is longer than the BC-based one due to the BI updates and more complex routing than the shortest path used by the BC-based algorithm. Notably, to make the solution time faster in ILP, we use $7 \%$ solution gap for the case of 100 demands.

\section{CONCLUSION}

This paper addressed the problem of joint VNF chain placement and routing, meeting their end-to-end delay require-

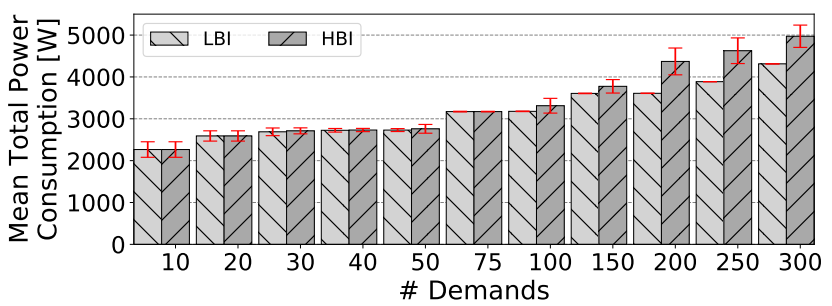

Fig. 6: Total power consumption for the HBI and LBI approaches. 
TABLE III: Mean runtime for different approaches (seconds)

\begin{tabular}{cccccccc}
\hline \#Demands & $\mathbf{1 0}$ & $\mathbf{2 0}$ & $\mathbf{3 0}$ & $\mathbf{4 0}$ & $\mathbf{5 0}$ & $\mathbf{7 5}$ & $\mathbf{1 0 0}$ \\
\hline ILP & 53.2 & 398.6 & 942.1 & 1742.1 & 2794.7 & 32269.3 & 18407 \\
\hline BI-Based & 0.102 & 0.149 & 0.187 & 0.238 & 0.263 & 0.373 & 0.405 \\
\hline BC-Based & 0.026 & 0.038 & 0.049 & 0.062 & 0.068 & 0.083 & 0.097 \\
\hline
\end{tabular}

ment, and also minimizing the overall power consumption by switches and PMs hosting the VNFs. We first proposed an ILP-based solution, and secondly two heuristics: (i) Blocking Islands (BI) abstraction based algorithm, and (ii) algorithm using the Betweenness Centrality (BC) property of a graph. The three solutions were compared in terms of the power consumption, the average demand's delay, the acceptance rate and the computation time. Our BI-based heuristic showed nearto-optimal results in terms of power consumption and an improvement in delay and computation time compared to the ILP, that is, the proposed BI-based heuristic is significantly faster and scales for large number of demands while reducing by $22 \%$ the average demand's delay, with a penalty of consuming $6 \%$ more power than the ILP solution. Furthermore, it was showed that choosing lower $\beta$ values (LBI) reduces the power consumption and increases the acceptance rate.

\section{ACKNOWLEDGEMENT}

This work was jointly supported under the Celtic-Plus subproject SEcure Networking for a DATa center cloud in Europe (SENDATE)-PLANETS (Project ID 16KIS0261, Celtic-Plus project ID C2015/3-1) funded by the German Federal Ministry of Education and Research (BMBF), and the Celtic-Plus subproject SENDATE-EXTEND funded by Vinnova (Celtic-Plus project ID C2015/3-3). This work was partly funded by the Swedish Research Council (VR) Framework project: Towards flexible and energy-efficient datacenter networks.

\section{REFERENCES}

[1] J. Halpern et al. RFC 7665: Service Function Chaining (SFC) Architecture. IETF Datatracker, 2015.

[2] ETSI Portal. Network Functions Virtualisation: An Introduction, Benefits, Enablers, Challenges and Call for Action, 2012.

[3] D. Abts et al. Energy proportional datacenter networks. In ACM SIGARCH Computer Architecture News, volume 38, pages 338-347. ACM, 2010.

[4] R. Bolla et al. Cutting the energy bills of internet service providers and telecoms through power management: An impact analysis. Computer Networks, 56(10):2320-2342, 2012.

[5] A. Gandhi et al. Optimal power allocation in server farms. In ACM SIGMETRICS Performance Evaluation Review, volume 37, pages 157168. ACM, 2009.

[6] P. Mahadevan et al. On energy efficiency for enterprise and data center networks. IEEE Communications Magazine, 49(8), 2011.

[7] B. Heller et al. ElasticTree: Saving Energy in Data Center Networks. In USENIX NSDI, volume 10, pages 249-264, 2010.

[8] Linton C Freeman. A set of measures of centrality based on betweenness. Sociometry, pages 35-41, 1977.

[9] H. Yao et al. Joint Optimization of VM Placement and Rule Placement towards Energy Efficient Software-Defined Data Centers. In Computer and Information Technology (CIT), 2016 IEEE International Conference on, pages 204-209. IEEE, 2016.

[10] H. Jin et al. Joint host-network optimization for energy-efficient data center networking. In Int. Symposium on Parallel \& Distributed Processing (IPDPS), pages 623-634. IEEE, 2013.

[11] A. Dalvandi et al. Application scheduling, placement, and routing for power efficiency in cloud data centers. IEEE Transactions on Parallel and Distributed Systems, 28(4):947-960, 2017.
[12] A. Mosa et al. Optimizing virtual machine placement for energy and SLA in clouds using utility functions. Jour. of Cloud Computing, 2016.

[13] Fabio Lopez-Pires and Benjamin Baran. Virtual machine placement literature review. arXiv preprint arXiv:1506.01509, 2015.

[14] A. Varasteh et al. Server consolidation techniques in Virtualized Data Centers: A survey. IEEE Systems Journal, 11(2):772-783, 2017.

[15] A. Hmaity et al. Virtual network function placement for resilient service chain provisioning. In Int. Workshop on Resilient Networks Design and Modeling (RNDM), pages 245-252. IEEE, 2016.

[16] D. Bhamare et al. A survey on Service Function Chaining. Journal of Network and Computer Applications, 75:138-155, 2016.

[17] W. Huang et al. An energy efficient virtual machine placement algorithm with balanced resource utilization. In Innovative Mobile and Internet Services in Ubiquitous Computing (IMIS), pages 313-319. IEEE, 2013

[18] W. Fang et al. VMPlanner: Optimizing virtual machine placement and traffic flow routing to reduce network power costs in cloud data centers. Computer Networks, 57(1):179-196, 2013.

[19] S. Kim et al. VNF-EQ: dynamic placement of virtual network functions for energy efficiency and QoS guarantee in NFV. Cluster Computing, 20(3):2107-2117, 2017.

[20] C. Pham et al. Traffic-aware and Energy-efficient vNF Placement for Service Chaining: Joint Sampling and Matching Approach. IEEE Transactions on Services Computing, 2017.

[21] N. Huin et al. Energy-efficient service function chain provisioning. JOCN, 10(3):114-124, 2018.

[22] K. Yang et al. Energy-Aware Service Function Placement for Service Function Chaining in Data Centers. In Global Communications Conference (GLOBECOM), 2016 IEEE, pages 1-6. IEEE, 2016.

[23] O. Soualah et al. Energy Efficient Algorithm for VNF Placement and Chaining. In Int. Symposium on Cluster, Cloud and Grid Computing (CCGRID), pages 579-588. IEEE/ACM, 2017.

[24] N. El Khoury et al. Energy-aware placement and scheduling of network traffic flows with deadlines on virtual network functions. In Int. Conf. on Cloud Networking (Cloudnet), pages 89-94. IEEE, 2016.

[25] V. Eramo et al. Migration energy aware reconfigurations of virtual network function instances in NFV architectures. IEEE Access, 5:49274938, 2017.

[26] A. Marotta et al. On the energy cost of robustness for green virtual network function placement in $5 \mathrm{~g}$ virtualized infrastructures. Computer Networks, 125:64-75, 2017.

[27] D. Boru et al. Energy-efficient data replication in cloud computing datacenters. Cluster Computing, 18(1):385-402, 2015.

[28] J.S. Chase et al. Managing energy and server resources in hosting centers. ACM SIGOPS operating systems review, 35(5):103-116, 2001.

[29] G. Chen et al. Energy-Aware Server Provisioning and Load Dispatching for Connection-Intensive Internet Services. In NSDI, volume 8, pages $337-350,2008$.

[30] A. Berl et al. Energy-efficient cloud computing. The computer journal, 53(7):1045-1051, 2010.

[31] Y. C. Lee et al. Energy efficient utilization of resources in cloud computing systems. The Journal of Supercomputing, 60:268-280, 2012.

[32] P. Vizarreta et al. QoS-driven function placement reducing expenditures in NFV deployments. In IEEE Int. Conf. on Communications, 2017.

[33] C. Frei et al. Simplifying network management using blocking island abstractions. Internal Note from the IMMuNe Project, 1997.

[34] T. Wang et al. Achieving energy efficiency in data centers using an artificial intelligence abstraction model. IEEE Trans. on Cloud Comp., 2015.

[35] S. Orlowski et al. SNDlib 1.0Survivable network design library. Networks, 55(3):276-286, 2010.

[36] A. Beloglazov et al. Energy-aware resource allocation heuristics for efficient management of data centers for cloud computing. Future generation computer systems, 28(5):755-768, 2012. 